\title{
A case for genotype-guided de-escalation of antiplatelet therapy after percutaneous coronary angioplasty
}

\author{
Larisa H Cavallari*,1 \& Craig R Lee ${ }^{2}$ \\ ${ }^{1}$ Department of Pharmacotherapy \& Translational Research, Center for Pharmacogenomics \& Precision Medicine, University of \\ Florida, Gainesville, FL 32610-0486, USA \\ ${ }^{2}$ Division of Pharmacotherapy \& Experimental Therapeutics, Eshelman School of Pharmacy \& McAllister Heart Institute, University \\ of North Carolina at Chapel Hill, Chapel Hill, NC 27599-7569, USA \\ *Author for correspondence: Tel.: +1 352273 8245; Icavallari@cop.ufl.edu
}

\section{"Genotyping may represent a more practical approach to guide de-escalation of antiplatelet therapy in the outpatient setting."}

First draft submitted: 2 April 2019; Accepted for publication: 9 April 2019; Published online: 6 August 2019

Keywords: antiplatelet • clopidogrel • CYP2C19 • genotype • percutaneous coronary intervention

\section{Antiplatelet therapy after percutaneous coronary intervention}

Dual antiplatelet therapy with aspirin plus a $\mathrm{P}_{2} \mathrm{Y}_{12}$ inhibitor (clopidogrel, ticagrelor or prasugrel) is indicated for at least 12 months following an acute coronary syndrome (ACS) and percutaneous coronary intervention (PCI) to prevent major adverse cardiovascular events (MACE) [1]. Prasugrel and ticagrelor exhibit more potent and consistent antiplatelet effects compared with clopidogrel, and were superior to clopidogrel in preventing MACE after an ACS and PCI in the TRITON TIMI-38 and PLATO trials, respectively [2,3]. As such, guidelines by the American College of Cardiology Foundation/American Heart Association state preference for these agents over clopidogrel following ACS and PCI [1]. However, prasugrel and ticagrelor are associated with a higher risk for noncoronary artery bypass graft-related major bleeding and higher cost compared with clopidogrel [2-4]. Ticagrelor use is also associated with dyspnea and gastrointestinal side effects [5]. These factors lead to higher discontinuation rates with newer agents and limit their widespread use $[4,5]$. Thus, while the use of prasugrel and ticagrelor has increased in recent years, clopidogrel remains commonly prescribed.

\section{CYP2C19 genotype \& clopidogrel response}

Clopidogrel is a prodrug that undergoes bioactivation via a two-step process to form its pharmacologically active metabolite that irreversibly binds to the platelet $\mathrm{P} 2 \mathrm{Y}_{12}$ receptor to inhibit platelet activation and subsequent aggregation. The cytochrome P450 (CYP) 2C19 enzyme is involved in both steps of the bioactivation process. The gene encoding CYP2C19 is highly polymorphic, with both no function and increased function alleles. The most common no function alleles are CYP2C19*2 (rs4244285, c.681G >A) and CYP2C19*3 (rs4986893, c.636G >A), occurring as a result of a splicing defect and premature stop codon, respectively. Other less common alleles with no or reduced function include ${ }^{*} 4-^{*} 8$. About $2-15 \%$ of individuals are poor metabolizers (PMs) with two no function alleles (e.g., ${ }^{*} 2{ }^{*} 2$ genotype) and no CYP2C19 enzyme activity [6]. Another $18-45 \%$ of individuals are intermediate metabolizers (IMs) with a single no function allele (e.g., ${ }^{*} 1 /{ }^{*} 2$ genotype) and significantly reduced enzyme activity. $5-30 \%$ of individuals have one or two ${ }^{*} 17$ alleles (e.g., ${ }^{*} 1 /{ }^{*} 17$ or ${ }^{*} 17 /{ }^{*} 17$ genotype) associated with increased gene transcription and increased enzyme activity. Persons of Asian ancestry have a higher frequency of no function alleles and a lower frequency of the increased function * 17 allele compared with persons of European or African ancestry.

Compared with patients without a no function allele, CYP2C19 PMs and IMs have lower concentrations of the active clopidogrel metabolite and less inhibition of platelet aggregation during clopidogrel treatment [7]. Multiple studies of patients receiving clopidogrel after PCI consistently show a higher risk for MACE in carriers of a no

Future $\because$ Medicine 
function allele compared with those without a no function allele [6,8]. The effect of CYP2C19 genotype outside the setting of PCI, as well as the effects of the ${ }^{*} 17$ allele, on clopidogrel effectiveness is less clear [6].

CYP2C19 genotype does not affect the pharmacokinetics, antiplatelet effects or clinical effectiveness of prasugrel or ticagrelor $[9,10]$. In clinical trials, the superiority of prasugrel and ticagrelor versus clopidogrel was greatest among CYP2C19 no function allele carriers [10,11]. In a genetic substudy of the TRITON-TIMI trial, prasugrel significantly reduced MACE risk compared with clopidogrel in patients with a CYP2C19 no function allele (hazard ratio [HR]: 0.57; 95\% CI: 0.39-0.83), but there was no difference in patients without a no function allele (HR: 0.98; $95 \%$ CI: $0.80-1.20$; genotype $\times$ treatment interaction; $p=0.046)[9,11]$. Similarly, in a genetic substudy of PLATO, ticagrelor more prominently reduced the 1-year risk for MACE compared with clopidogrel in no function allele carriers (HR: 0.77; 95\% CI: 0.60-0.99) than in those without a no function allele (HR: 0.86; 95\% CI: 0.74-1.01), though the genotype $\times$ treatment interaction was nonsignificant $[10]$.

\section{Clopidogrel labeling change \& pharmacogenetic guidelines}

In 2010, the US FDA approved revision of the clopidogrel label to include a boxed warning about reduced clopidogrel effectiveness in CYP2C19 PMs [12]. Multiple studies have shown that clopidogrel-treated IMs are also at increased risk for MACE [6,8]. The Clinical Pharmacogenetics Implementation Consortium recommends use of prasugrel or ticagrelor in patients known to have one or two CYP2C19 no function alleles, but does not address whether genetic testing should be performed [6]. The American College of Cardiology Foundation/American Heart Association PCI guidelines recommend against routine genotyping citing a lack of clinical trial data, but state that CYP2C19 testing and use of prasugrel or ticagrelor in high-risk patients genetically predisposed to poor clopidogrel response 'might be considered' (Class IIb, Level of Evidence: C) [1].

\section{Clinical implementation of CYP2C19 genotyping}

CYP2C19 testing is now part of clinical practice to guide antiplatelet therapy after PCI at the University of Florida Health (FL, USA), University of North Carolina at Chapel Hill (NC, USA) and multiple other institutions across the USA [13]. At the time our institutions began clinical genotyping, clopidogrel was the first-line $\mathrm{P}_{2} \mathrm{Y}_{12}$ inhibitor, and genotype results were used to selectively 'escalate' therapy from clopidogrel to prasugrel or ticagrelor in no function allele carriers; noncarriers usually remained on clopidogrel [13]. A multisite analysis of outcomes with genotype-guided antiplatelet therapy after PCI on behalf of the NHGRI-funded Implementing GeNomics In pracTicE (IGNITE) Network showed that genotyping for CYP2C19, and prescribing prasugrel or ticagrelor instead of clopidogrel in no function allele carriers, resulted in significantly lower risk for MACE [14]. In contrast, there was no difference in outcome between no function allele carriers treated with prasugrel or ticagrelor and those without a no function allele, who were predominately treated with clopidogrel. The evidence base supporting the use of CYP2C19 genotyping to guide antiplatelet therapy selection after PCI continues to expand [15]. The ongoing TAILOR-PCI (NCT01742117) and POPULAR-Genetics (NCT01761786) randomized clinical trials are further examining the impact of genotype-guided escalation on clinical outcomes.

\section{Emergence of de-escalation of antiplatelet therapy}

The greater bleeding risk and cost with prasugrel and ticagrelor compared with clopidogrel are most evident during chronic therapy [2-4]. A strategy of using more potent agents early after PCI when risk for atherothrombotic events is highest, following by de-escalation to clopidogrel has been proposed as a means of maximizing benefit and minimizing risk of bleeding, treatment discontinuation and cost [16]. De-escalation is becoming more common in practice; however, there are conflicting outcomes data with an empiric (nonguided) de-escalation approach, with very early de-escalation to clopidogrel after ACS increasing risk of recurrent atherothrombotic events $[17,18]$.

On the other hand, the recent TROPICAL-ACS trial showed that a de-escalation approach guided by platelet function testing (PFT) was noninferior to universal prasugrel at preventing MACE over 12 months, with a nonsignificant trend toward fewer clinically significant bleeding events [19]. These data were recently integrated into the 2018 European Society of Cardiology PCI guidelines, indicating that de-escalation of dual antiplatelet therapy (e.g., switch from prasugrel or ticagrelor to clopidogrel) guided by PFT may be considered as an alternative antiplatelet therapy strategy, especially for ACS patients deemed unsuitable for 12 months of prasugrel or ticagrelor (Class IIb, Level of Evidence: B) [20]. A major limitation with PFT, however, is that it must be performed while the patient is on treatment. Patients randomized to the de-escalation arm in TROPICAL-ACS were treated with prasugrel for 7 days, switched to clopidogrel for 7 days, and then evaluated by PFT. The subset of patients with high 
on-treatment platelet reactivity (i.e., clopidogrel nonresponders) were switched back to prasugrel for the duration of treatment. Such a strategy may not be practical in a real-world setting.

\section{Future perspective}

Genotyping may represent a more practical approach to guide de-escalation of antiplatelet therapy in the outpatient setting. Genotyping, in contrast to PFT, can be done a priori, without the need to switch the patient from prasugrel or ticagrelor to clopidogrel to make a decision. Use of a genotype-guided de-escalation strategy would involve initiating patients on prasugrel or ticagrelor after PCI, at which time a sample may be collected for genetic analysis. Genotype results could then become available within the initial weeks of PCI during treatment with a more potent antiplatelet therapy, in contrast to an escalation strategy that requires centers to have genetic testing capacity onsite and prompt availability of the genetic test result after PCI. Prasugrel or ticagrelor could be continued for patients with a CYP2C19 no function allele, who would be expected to respond poorly to clopidogrel. However, for approximately $70 \%$ of patients without a no function allele, clopidogrel would be expected to be effective. Thus, for patients at high bleeding risk or unable to tolerate or afford prasugrel or ticagrelor, the physician would have more precise assurance that clopidogrel should be an effective antiplatelet therapy. An examination of outcomes with genotype-guided de-escalation of antiplatelet therapy after PCI in large, multicenter populations is needed to determine the clinical utility of this emerging precision antiplatelet therapy strategy.

\section{Author contributions}

LH Cavallari drafted the manuscript and CR Lee drafted the manuscript.

\section{Disclaimer}

The content is solely the responsibility of the authors and does not necessarily represent the official views of the NIH.

Financial \& competing interests disclosure

Work by LHC is supported by NIH/NHGRI (U01 HG 007269) and NIH/NCATS (UL1 TR 001427). The authors have no other relevant affiliations or financial involvement with any organization or entity with a financial interest in or financial conflict with the subject matter or materials discussed in the manuscript apart from those disclosed.

No writing assistance was utilized in the production of this manuscript.

\section{References}

1. Levine GN, Bates ER, Blankenship JC et al. 2015 ACC/AHA/SCAI focused update on primary percutaneous coronary intervention for patients with ST-elevation myocardial infarction: an update of the 2011 ACCF/AHA/SCAI guideline for percutaneous coronary intervention and the $2013 \mathrm{ACCF} / \mathrm{AHA}$ guideline for the management of ST-elevation myocardial infarction: a report of the American College of Cardiology/American Heart Association Task Force on clinical practice guidelines and the society for cardiovascular angiography and interventions. Circulation 133(11), 1135-1147 (2016).

2. Wiviott $\mathrm{SD}$, Braunwald E, McCabe $\mathrm{CH}$ et al. Prasugrel versus clopidogrel in patients with acute coronary syndromes. N. Engl. J. Med. 357(20), 2001-2015 (2007).

3. Wallentin L, Becker RC, Budaj A et al. Ticagrelor versus clopidogrel in patients with acute coronary syndromes. N. Engl. J. Med. 361(11), 1045-1057 (2009).

4. Dayoub EJ, Seigerman M, Tuteja $S$ et al. Trends in platelet adenosine diphosphate P2Y12 receptor inhibitor use and adherence among antiplatelet-naive patients after percutaneous coronary intervention, 2008-2016. JAMA Intern. Med. 178(7), 943-950 (2018).

5. Zanchin T, Temperli F, Karagiannis A et al. Frequency, reasons, and impact of premature ticagrelor discontinuation in patients undergoing coronary revascularization in routine clinical practice: results from the Bern Percutaneous Coronary Intervention Registry. Circ. Cardiovasc. Interv. 11(5), e006132 (2018).

6. Scott SA, Sangkuhl K, Stein CM et al. Clinical Pharmacogenetics Implementation Consortium guidelines for CYP2C19 genotype and clopidogrel therapy: 2013 update. Clin. Pharmacol. Ther. 94(3), 317-323 (2013).

7. Mega JL, Close SL, Wiviott SD et al. Cytochrome p-450 polymorphisms and response to clopidogrel. N. Engl. J. Med. 360(4), 354-362 (2009).

8. Mega JL, Simon T, Collet JP et al. Reduced-function CYP2C19 genotype and risk of adverse clinical outcomes among patients treated with clopidogrel predominantly for PCI: a meta-analysis. JAMA 304(16), 1821-1830 (2010).

9. Mega JL, Close SL, Wiviott SD et al. Cytochrome p450 genetic polymorphisms and the response to prasugrel: relationship to pharmacokinetic, pharmacodynamic, and clinical outcomes. Circulation 119(19), 2553-2560 (2009). 
10. Wallentin L, James S, Storey RF et al. Effect of CYP2C19 and ABCB1 single nucleotide polymorphisms on outcomes of treatment with ticagrelor versus clopidogrel for acute coronary syndromes: a genetic substudy of the PLATO trial. Lancet 376(9749), 1320-1328 (2010).

11. Sorich MJ, Vitry A, Ward MB, Horowitz JD, McKinnon RA. Prasugrel vs. clopidogrel for cytochrome p450 2C19-genotyped subgroups: integration of the TRITON-TIMI 38 trial data. J. Thromb. Haemost. 8(8), 1678-1684 (2010).

12. Holmes DR Jr, Dehmer GJ, Kaul S, Leifer D, O'Gara PT, Stein CM. ACCF/AHA clopidogrel clinical alert: approaches to the FDA "boxed warning": a report of the American College of Cardiology Foundation Task Force on clinical expert consensus documents and the American Heart Association endorsed by the Society for Cardiovascular Angiography and Interventions and the Society of Thoracic Surgeons. J. Am. Coll. Cardiol. 56(4), 321-341 (2010).

13. Empey PE, Stevenson JM, Tuteja $S$ et al. Multisite investigation of strategies for the implementation of CYP2C19 genotype-guided antiplatelet therapy. Clin. Pharmacol. Ther. 104(4), 664-674 (2018)

14. Cavallari LH, Lee CR, Beitelshees AL et al. Multi-site investigation of outcomes with implementation of CYP2C19 genotype-guided antiplatelet therapy after percutaneous coronary intervention. JACC Cardiovasc. Interv. 11(2), 181-191 (2018).

15. Klein MD, Lee CR, Stouffer GA. Clinical outcomes of CYP2C19 genotype-guided antiplatelet therapy: existing evidence and future directions. Pharmacogenomics 19(13), 1039-1046 (2018).

16. Angiolillo DJ, Rollini F, Storey RF et al. International expert consensus on switching platelet P2Y12 receptor-inhibiting therapies. Circulation 136(20), 1955-1975 (2017).

17. Cuisset T, Deharo P, Quilici J et al. Benefit of switching dual antiplatelet therapy after acute coronary syndrome: the TOPIC (timing of platelet inhibition after acute coronary syndrome) randomized study. Eur. Heart J. 38(41), 3070- 3078 (2017).

18. De Luca L, D’Ascenzo F, Musumeci G et al. Incidence and outcome of switching of oral platelet P2Y12 receptor inhibitors in patients with acute coronary syndromes undergoing percutaneous coronary intervention: the SCOPE registry. EuroIntervention 13(4), 459-466 (2017).

19. Sibbing D, Aradi D, Jacobshagen C et al. Guided de-escalation of antiplatelet treatment in patients with acute coronary syndrome undergoing percutaneous coronary intervention (TROPICAL-ACS): a randomised, open-label, multicentre trial. Lancet 390(10104), 1747-1757 (2017)

20. Neumann FJ, Sousa-Uva M, Ahlsson A et al. 2018 ESC/EACTS guidelines on myocardial revascularization. Eur. Heart J. 40(2), 87-165 (2019). 